${ }^{1}$ Unidad de Medicina Basada en Evidencia, Facultad de Medicina, Pontificia Universidad Católica de Chile.

${ }^{2}$ Departamento de Medicina Interna, Facultad de Medicina, Pontificia Universidad Católica de Chile. Santiago de Chile. ${ }^{3}$ Unidad Docente Asociada, Hospital Dr. Sótero del Río.

Correspondencia a: Dr. Gabriel Rada. E-mail: umbeuc@med. puc.cl

\section{Análisis crítico de un artículo: colonografía por tomografía computada tuvo buen rendimiento para la pesquisa de cáncer colorrectal en pacientes de alto riesgo}

\author{
MARIO CABEZÓN ${ }^{1}$, GABRIEL RADA ${ }^{1,2,3}$ \\ Critically appraised article
}

Regge D, Laudi C, Galatola G. Diagnostic Accuracy of Computed Tomographic Colonography for the Detection of Advanced Neoplasia in Individuals at Increased Risk of Colorectal Cancer. JAMA 2009: 301(23): 2453-61

Context: Computed tomographic (CT) colonography has been recognized as an alternative for colorectal cancer (CRC) screening in average-risk individuals, but less information is available on its performance in individuals at increased risk of CRC. Objective: To assess the accuracy of CT colonography in detecting advanced colorectal neoplasia in asymptomatic individuals at increased risk of CRC using unblinded colonoscopy as the reference standard. Design, Setting, and Participants: This was a multicenter, cross-sectional study. Individuals at increased risk of CRC due to either family history of advanced neoplasia in first-degree relatives, personal history of colorectal adenomas, or positive results from fecal occult blood tests (FOBTs) were recruited in 11 Italian centers and 1 Belgian center between December 2004 and May 2007. Each participant underwent CT colonography followed by colonoscopy on the same day. Main Outcome Measures: Sensitivity and specificity of CT colonography in detecting individuals with advanced neoplasia (ie, advanced adenoma or CRC) $6 \mathrm{~mm}$ or larger. Results: Of 1103 participants, 937 were included in the final analysis: 373 cases in the family-history group, 343 in the group with personal history of adenomas, and 221 in the FOBT-positive group. Overall, CT colonography identified 151 of 177 participants with advanced neoplasia 6 mm or larger (sensitivity, 85.3\%; 95\% confidence interval [CI], 79.0\%-90.0\%) and correctly classified results as negative for 667 of 760 participants without such lesions (specificity, 87.8\%; 95\% CI, 85.2\%-90.0\%). The positive and negative predictive values were $61.9 \%$ (95\% CI, 55.4\%-68.0\%) and $96.3 \%$ (95\% CI, 94.6\%-97.5\%), respectively; after group stratifcation, a significantly lower negative predictive value was found for the FOBT-positive group (84.9\%; 95\% CI, 76.2\%-91.3\%; $\left.P_{-} .001\right)$. Conclusions In a group of persons at increased risk for CRC, CT colonography compared with colonoscopy resulted in a negative predictive value of $96.3 \%$ overall. When limited to FOBT-positive persons, the negative predictive value was $84.9 \%$.

(Rev Med Chile 2011; 139: 676-680). 


\section{Conclusión de los revisores}

Una colonografía por tomografía computada (TC) normal tuvo un buen rendimiento para descartar el cáncer colorrectal (CCR) en pacientes asintomáticos con historia familiar de CCR o polipectomía previa. En pacientes con un test de hemorragia oculta positiva previa, el rendimiento del examen no sería tan bueno.

\section{Pregunta clínica}

Comparada con la colonoscopía, ¿es útil la colonografía por tomografía computada para la detección de CCR avanzado en pacientes asintomáticos con factores de riesgo?

\section{Introducción}

El CCR causa 210.000 muertes al año en Europa ${ }^{1}$. La mayoría de las lesiones provienen de un pólipo adenomatoso originado en el epitelio colónico, y la polipectomía disminuye el riesgo de transformación maligna y de mortalidad por $\mathrm{CCR}^{2}$.

Los pacientes con riesgo aumentado son aquellos que presentan el antecedente de CCR en un familiar de primer grado, una polipectomía previa o un test de hemorragia oculta positivo. Si bien hay controversia acerca del mejor método de tamizaje en población general, en este grupo existe relativo consenso en que el examen más indicado es la colonoscopía, pero la adherencia a este procedimiento sigue siendo subóptima ${ }^{3,4}$.

Una alternativa menos invasiva y mejor tolerada que la colonoscopía es la colonografía por TC. En algunos estudios ha demostrado buen rendimiento para la detección de $\mathrm{CCR}^{5,6}$, siendo actualmente recomendada como método de tamizaje en la población general por algunas sociedades científicas ${ }^{7}$. La evidencia sobre el rendimiento de este examen en pacientes de alto riesgo aun no está precisada.

\section{Características del estudio}

Tipo de estudio: Estudio clínico de corte transversal sobre la exactitud diagnóstica de un test (diagnostic test accuracy), multicéntrico, realizado en 10 hospitales de Italia y 1 de Bélgica, entre diciembre de 2004 y mayo de 2007.

Pacientes: Fueron incluidos 937 pacientes con factores de riesgo para CCR, divididos en 3 grupos.

Grupo 1: 373 pacientes entre 45 y 60 años con historia de un familiar de primer grado con CCR.

Grupo 2: 343 pacientes entre 18 y 70 años con historia personal de resección de adenomas.

Grupo 3: 221 pacientes entre 59 y 69 años con test de hemorragia oculta positivo.

Intervención: Test diagnóstico: Los pacientes fueron sometidos a una colonografía por TC y posteriormente a una colonoscopía el mismo día, en el mismo centro (estándar de oro o gold standard). El informe de la colonografía por TC fue divido en 6 segmentos colónicos por separado. Sólo al finalizar el informe de cada segmento de la endoscopía, el endoscopista era informado del resultado de la colonografía por TC.

Se consideró como diagnóstico positivo en la TC la presencia de una lesión mayor 
o igual a $6 \mathrm{~mm}$. La colonoscopía ( gold standard) clasificaba posteriormente esa lesión como un verdadero positivo o falso positivo. Si la lesión era menor a $6 \mathrm{~mm}$, se consideraba colonografía por TC negativa, la cual era clasificada como verdadero negativo o falso negativo en la colonoscopía.

Objetivo: Evaluar el rendimiento de la colonografía por TC en comparación con la colonoscopía, en términos de sensibilidad, especificidad y valores predictivos. Los likelihood ratios han sido calculados por los autores de este análisis crítico.

\section{Validez (riesgo de sesgo) del estudio:}

1. ¿Se incluyó a un espectro apropiado de pacientes? Sí

2. ¿El gold standard fue adecuado? Sí

3. ¿El test fue evaluado sin conocer el resultado del gold standard y viceversa (ciego)? Parcialmente

4. ¿El gold standard fue independiente del test? Sí

5. ¿Se realizó el gold standard independiente del resultado del test? Sí

\section{Resultados}

\begin{tabular}{|c|c|c|c|c|}
\hline & $\begin{array}{l}\text { Sensibilidad } \\
\text { (IC 95\%) }\end{array}$ & $\begin{array}{l}\text { Especificidad } \\
\text { (IC 95\%) }\end{array}$ & $\begin{array}{l}\text { Likelihood ratio si la TC } \\
\text { es positiva (IC 95\%) }\end{array}$ & $\begin{array}{c}\text { Likelihood ratio si la TC } \\
\text { es negativa (IC 95\%) }\end{array}$ \\
\hline Global & $\begin{array}{c}85,3 \\
(79 \text { a } 90)\end{array}$ & $\begin{array}{c}87,8 \\
(85,2 \text { a } 90)\end{array}$ & $\begin{array}{c}6,97 \\
(5,70 \text { a } 8,51)\end{array}$ & $\begin{array}{c}0,17 \\
(0,12 \text { a } 0,23)\end{array}$ \\
\hline Historia Familiar & $\begin{array}{c}82,1 \\
(62,4 \text { a } 93,2)\end{array}$ & $\begin{array}{c}93,6 \\
(90,4 \text { a } 95,9)\end{array}$ & $\begin{array}{c}12 \\
(7,69 \text { a } 18)\end{array}$ & $\begin{array}{c}0,19 \\
(0,09 \text { a } 0,42)\end{array}$ \\
\hline Resección previa & $\begin{array}{c}84,2 \\
(68,1 \text { a } 93,4)\end{array}$ & $\begin{array}{c}85,3 \\
(80,7 \text { a } 88,9)\end{array}$ & $\begin{array}{c}5,6 \\
(4,14 \text { a } 7,57)\end{array}$ & $\begin{array}{c}0,19 \\
(0,09 \text { a } 0,39)\end{array}$ \\
\hline $\begin{array}{l}\text { Antecedente de } \\
\text { test de hemorragia } \\
\text { oculta positivo }\end{array}$ & $\begin{array}{c}86,5 \\
(78,4 \text { a } 92)\end{array}$ & $\begin{array}{c}76,4 \\
(67,1 \text { a } 83,7)\end{array}$ & $\begin{array}{c}3,58 \\
(2,53 \text { a } 5,06)\end{array}$ & $\begin{array}{c}0,18 \\
(0,11 \text { a } 0,29)\end{array}$ \\
\hline
\end{tabular}

$\mathrm{IC}=$ Intervalo de confianza

\section{Comentarios}

\section{Comentarios acerca de la validez (riesgo de sesgo):}

- Se trata de un estudio que evalúa el rendimiento de un test como método de tamizaje, a través de un estudio transversal. Se incluyó un espectro apropiado de pacientes, con factores de riesgo para CCR, en quienes existe incertidumbre diagnóstica.

- Con respecto al gold standard, la colonoscopía es reconocida como tal para el diagnóstico de CCR. Se consideraron positivas aquellas lesiones mayores a $6 \mathrm{~mm}$, siendo este parámetro el más usado por los estudios internacionales, por su fuerte asociación con riesgo de CCR 5 . El gold standard se realizó a todos los pacientes del estudio, de forma independiente, sin importar el resultado de la colonografía por TC.

- Uno de los aspectos discutibles, es que si bien el diseño incluía a la colonografía de forma "ciega", el resultado estaba en la manos del endoscopista al final de cada 
segmento colónico analizado (por eso la definimos como "parcialmente"). Es razonable pensar que algunos endoscopistas vieran el resultado antes, simultáneamente a la realización de la colonoscopía. Es difícil asegurar que se mantuvo el ciego en esas circunstancias, en el 100\% de los pacientes.

\section{Comentarios acerca de los resultados}

El test muestra resultados aceptables de sensibilidad y especificidad. Si utilizamos los LR obtenidos podemos evaluar como una TC modifica la probabilidad; si consideramos la prevalencia global (19\%) de CCR que obtuvieron los autores (probabilidad pretest), una colonografía por TC positiva $(\mathrm{LR}=7)$, aumenta la probabilidad post test de tener CCR a $62 \%$, mientras que una TC negativa $(L R=0,17)$ la reduce a $4 \%$.

Si realizamos el mismo ejercicio para el subgrupo con test de hemorragia oculta positivo, cuya prevalencia es más alta (50\%), y en donde el rendimiento del test fue menor, se obtienen los siguientes resultados: un test positivo aumenta la probabilidad post test a $78 \%$ y uno negativo la disminuye a $15 \%$.

Con respecto a los pacientes con historia familiar de CCR o polipectomía, estudios observacionales muestran que tienen prevalencias más bajas (aproximadamente $8 \%)^{8}$. Si aplicáramos el test para el subgrupo de historia familiar, un examen positivo aumenta la probabilidad a $52 \%$, mientras que un examen negativo la disminuye a $2 \%$, siendo este un escenario frecuente en la práctica clínica.

Es muy importante considerar que todos los resultados sobre el rendimiento del test reflejan el estimador que más probablemente corresponde a la realidad. Sin embargo, al igual que en cualquier estudio, es fundamental fijarse en el intervalo de confianza. En este estudio los resultados son bastante precisos, y los valores de los intervalos no modifican sustancialmente la conducta clínica. Por ejemplo, si consideramos el LR si la TC es positiva (6,97; IC 95\% 5,70 a 8,51); tanto si el valor real fuera 5,7 como si fuera 8,5 (post test de 57 vs $66 \%$ respectivamente), probablemente nuestra conducta sería la misma.

\section{Comentarios acerca de la aplicabilidad}

El CCR es una patología de gran importancia en Chile, con un aumento estable en los últimos años ${ }^{9,10}$. El uso de la colonografía por TC ha tenido un aumento progresivo en países de altos ingresos para la pesquisa de CCR y en nuestro medio, son cada vez más los centros que cuentan con este examen. Este estudio muestra que en los pacientes de alto riesgo, la colonografía por TC podría constituir un test que permita bajar suficientemente la probabilidad, como para reemplazar a la colonoscopía convencional. Es importante enfatizar que la decisión acerca de cuándo la probabilidad post test es suficientemente baja como para descartar la patología (umbral de estudio adicional), depende de múltiples factores, y debe ajustarse a cada caso ${ }^{11}$.

En el caso de los pacientes con un test de hemorragias ocultas positivo, su alta probabilidad pre test hace que un test negativo no baje suficientemente la probabilidad de CCR, por lo que de confirmarse los resultados de este estudio, parece ser que este examen no constituiría una buena opción en este subgrupo.

Es importante destacar que no obstante los promisorios resultados de este estudio, sería importante contar con un estudio clínico randomizado que demuestre impacto sobre la mortalidad u otro outcome importante para el paciente. Las razones por la cual este aspecto es relevante se mencionan con mayor detalle en el artículo publicado en este mismo número de la revista, sobre la aplicabilidad de un test diagnóstico ${ }^{12}$.

La colonografía por TC tiene la ventaja de ser rápida y bien tolerada. Su tasa de efectos adversos es mínima y además permite evaluar estructuras extracolónicas. Algunas de sus indicaciones más comunes se dan en pacientes que no pudieron tolerar 
Colonografía por TAC en pesquisa de cáncer de colon- M. Cabezón et al

una colonoscopía o cuando lesiones estenosantes no permiten evaluar el colon distal ${ }^{13}$. En ese sentido, la colonoscopía tiene una tasa de complicación menor al 1\%, siendo la más frecuente el sangrado post polipectomía ${ }^{14}$.

Las desventajas de la colonoscopía por TC son su alto costo, por el momento, en Chile (entre 385.000 y 455.000 pesos en centros privados), su baja disponibilidad, sus requerimientos técnicos (software, personal entrenado), el hecho de no poder tomar biopsias o realizar procedimientos, además de la irradiación al paciente ${ }^{13}$.

Es importante que los clínicos tengan más conocimiento y acceso a este examen, de manera que sea explicado a los pacientes y puedan tomar una decisión en conjunto con ellos, intentando así mejorar la adherencia a los métodos de tamizaje y disminuir las tasas de mortalidad del CCR en Chile.

\section{Referencias}

1. Ferlay J, Autier P, Boniol M, Heanue M, Colombet M, Boyle P. Estimates of the cancer incidence and mortality in Europe in 2006. Ann Oncol 2007; 18: 581-92.

2. Winawer SJ, Zauber AG, Ho MN, O’brien MJ, Gottlieb LS, Sternberg SS, et al. Prevention of colorectal cancer by colonoscopy polypectomy: the National Polyp Study Work Group. N Engl J Med 1993; 329: 1977-81.

3. Bujanda L, Sarasqueta C, Zubiaurre L, Cosme A, Muñoz C, Sánchez A et al; EPICOLON Group. Low adherence to colonoscopy in the screening of first-degree relatives of patients with colorectal cancer. Gut 2007; 56: 1714-8.

4. Nadel MR, Shapiro JA, Klabunde CN, Seeff LC, Uhler $\mathrm{R}$, Smith RA, et al. A national survey of primary care physicians' methods for screening for fecal occult blood. Ann Intern Med 2005; 142: 86-94.

5. Pickhardt PJ, Choi JR, Hwang I, Butler JA, Puckett ML, Hildebrandt HA, et al. Computed tomographic virtual colonoscopy to screen for colorectal neoplasia in asymptomatic adults. N Engl J Med 2003; 349: 2191-200.

6. Johnson CD, Chen MH, Toledano AY, Heiken JP, Dachman A, Kuo MD, et al. Accuracy of CT colonography for detection of large adenomas and cancers. N Engl J Med 2008; 359: 1207-17.

7. Levin B, Lieberman DA, McFarland B, Andrews KS, Brooks D, Bond J, et al. Screening and surveillance for the early detection of colorectal cancer and adenomatous polyps, 2008: a joint guideline from the American Cancer Society, the US Multi-Society Task Force on Colorectal Cancer, and the American College of Radiology. Gastroenterology 2008; 134: 1570-95.

8. Cottet V, Pariente A, Nalet B, Lafon J, Milan C, Olschwang S, et al. Prevalence of nonpolypoid (flat and depressed) colorectal neoplasms in asymptomatic and symptomatic adults. Gastroenterology 2007; 133: 108692.

9. Donoso A, Villarroel L, Pinedo G. Aumento de la mortalidad por cáncer de colon en Chile, 1990-2003. Rev Med Chile 2006; 134: 152-8.

10. Minsal. Mortalidad por causas especificas 1990-2005. http://deis.minsal.cl/deis/indexmc2.asp [Consultado el 28 de Noviembre de 2010].

11. Capurro D, Rada G. El proceso diagnóstico. Rev Med Chile 2007; 135: 530-4.

12. Rivera S, Letelier LM. Aplicabilidad de un estudio sobre test diagnósticos. Rev Med Chile (en prensa) OJO puede que esté precediendo en este número.

13. Fernández M, Aldana H. Colonoscopía Virtual. Rev Chil Radiol 2006; 12: 64-9.

14. Wilkins T, LeClair B, Smolkin M, Davies K, Thomas A, Taylor ML, et al. Screening colonoscopies by primary care physicians: a meta-analysis. Ann Fam Med 2009; 7: 56-62. 